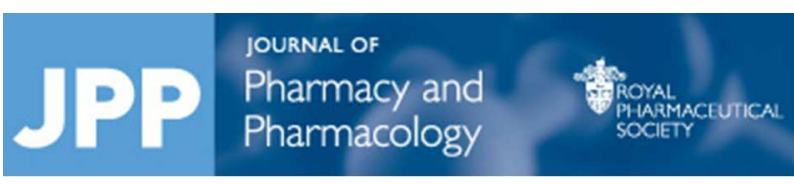

\title{
Development and optimization of ketoconazole oral strips by means of continuous hot-melt extrusion processing
}

\begin{tabular}{|c|c|}
\hline Journal: & Journal of Pharmacy and Pharmacology \\
\hline Manuscript ID & JPP-15-0735.R1 \\
\hline Wiley - Manuscript type: & Research Paper \\
\hline Date Submitted by the Author: & $n / a$ \\
\hline Complete List of Authors: & $\begin{array}{l}\text { Maniruzzaman, Mohammed; University of Sussex School of Life Sciences } \\
\text { Farias, Smirna; University of Greenwich, School of Science } \\
\text { Boateng, Joshua; University of Greenwich, Faculty of Engineering and } \\
\text { Science } \\
\text { Slipper, Ian; University of Greenwich, School of Science } \\
\text { Chowdhry, Babur; University of Greenwich, School of Science } \\
\text { Nair, Arun; Fuji Chemical Industry Co., Ltd., Shibakoen, Minato-ku, } \\
\text { 1050011 } \\
\text { Douroumis, Dennis; University of Greenwich, Medway School of Science }\end{array}$ \\
\hline Keywords: & $\begin{array}{l}\text { Dosage Form Design and Characterisation }<\text { Pharmaceutics and Drug } \\
\text { Delivery, Controlled and Sustained Release Systems < Pharmaceutics and } \\
\text { Drug Delivery, Development of Novel Analytical Techniques }< \\
\text { Pharmaceutical Analysis }\end{array}$ \\
\hline Abstract: & $\begin{array}{l}\text { Objectives The aim of this study was to develop mucoadhesive oral strips } \\
\text { using hot-melt extrusion as a continuous manufacturing process. } \\
\text { Methods Powder blends of ketoconazole, a water insoluble drug; either } \\
\text { hydroxypropyl methylcellulose (HPMC) or soluplus (SOL), sorbitol (SRB) } \\
\text { and magnesium aluminometasilicate (MAS) were extruded to manufacture } \\
\text { thin strips with } 0.5 \text { mm thickness. The presence of the inorganic } \\
\text { metasilicate facilitated smooth processing of the extruded strips as it } \\
\text { worked as an absorbent directly impacting on the extensive mixing of the } \\
\text { drug/excipients inside the extruder barrel. } \\
\text { Key findings The use of MAS also favoured the rapid hydration, swelling } \\
\text { and eventual disintegration of the strips. Differential scanning calorimetry } \\
\text { (DSC) and transmission X-ray diffraction (TXRD) analysis revealed the } \\
\text { existence of the amorphous drug within the extruded strips. Scanning } \\
\text { electron microscopy (SEM) and energy dispersive X-Ray (EDS) undertaken } \\
\text { on the formulations showed a homogeneous drug distribution within the } \\
\text { extruded strips. } \\
\text { Conclusion The strips produced via continuous HME processing showed } \\
\text { significantly faster release of KTZ compared to the bulk drug substance. }\end{array}$ \\
\hline
\end{tabular}


SCHOLARONE ${ }^{\text {'m }}$

Manuscripts 


\section{Development and optimization of ketoconazole oral strips by means of continuous hot-melt extrusion processing}

Mohammed Maniruzzaman ${ }^{1 *}$, Smirna Farias ${ }^{2}$, Ian J. Slipper ${ }^{2}$, Joshua S. Boateng ${ }^{2}$, Babur Z.Chowdhry ${ }^{2}$, Arun Nair ${ }^{3}$, Dennis Douroumis $^{2 *}$

${ }^{1}$ Department of Pharmacy (Chemistry), School of Life Sciences, University of Sussex, Falmer, Brighton, BN1 9QT

${ }^{2}$ Faculty of Engineering and Science, School of Science, University of Greenwich, Chatham Maritime, Chatham, Kent ME4 4TB, UK

${ }^{2}$ Fuji Chemical Industry Co., Ltd., Shibakoen, Minato-ku, Tokyo 1050011, Japan.

*Correspondence: Mohammed Maniruzzaman or Dennis Douroumis, email: D.Douroumis@gre.ac.uk; M.Maniruzzaman@sussex.ac.uk, Phone: +44 208331 8440/+44 1273 877639, Fax: 0044 (0) 208331 9805. 


\section{ABSTRACT}

Objectives The aim of this study was to develop mucoadhesive oral strips using hotmelt extrusion as a continuous manufacturing process.

Methods Powder blends of ketoconazole, a water insoluble drug; either hydroxypropyl methylcellulose (HPMC) or soluplus (SOL), sorbitol (SRB) and magnesium aluminometasilicate (MAS) were extruded to manufacture thin strips with $0.5 \mathrm{~mm}$ thickness. The presence of the inorganic metasilicate facilitated smooth processing of the extruded strips as it worked as an absorbent directly impacting on the extensive mixing of the drug/excipients inside the extruder barrel.

Key findings The use of MAS also favoured the rapid hydration, swelling and eventual disintegration of the strips. Differential scanning calorimetry (DSC) and transmission X-ray diffraction (TXRD) analysis revealed the existence of the amorphous drug within the extruded strips. Scanning electron microscopy (SEM) and energy dispersive X-Ray (EDS) undertaken on the formulations showed a homogeneous drug distribution within the extruded strips.

Conclusion The strips produced via continuous HME processing showed significantly faster release of KTZ compared to the bulk drug substance.

Keywords: Continuous manufacturing, ketoconazole, oral strips, energy dispersive X-Ray, surface analysis, amorphous. 


\section{Introduction}

'Strips' (commonly referred to as films) can be defined as thin sheets containing one or more polymers with or without a plasticizer and can effectively be used as a drug delivery system. Whilst, the solvent - casting approach - currently a pharmaceutically adopted process, allows better uniformity, flexibility and adjustable thickness, this approach is limited by low drug loading, high costs and time consumption for development. ${ }^{[1-3]}$ Furthermore, the excessive use of various solvents to dissolve insoluble polymer or drug makes the solvent casting method questionable from the regulatory viewpoint. Moreover, the hazardous nature of most organic solvents and the residual solvents ${ }^{[4-7]}$ left in the films may create significant environmental and health concerns. Therefore, there is increasing current interest in alternative technologies in the pharmaceutical industry to overcome some or all of the challenges currently associated with solvent film casting.

Hot melt extrusion (HME) has been successfully exploited for the manufacturing of oral thin films or strips due to its unique advantages. ${ }^{[8]} \mathrm{HME}$ is a solvent free green technology, easy to scale up and operates in a continuous mode.

Another key advantage of HME is that robust extruded films/strips can be obtained in a single processing step making the process economically viable. In a HME process, melt extruded strips are produced via feeding the blends of appropriate amounts of the relevant polymer(s), drug, plasticiser and other excipients into the preheated extruder barrel and conveying them with rotating screws into homogenous sheets. Other excipients can be added to enhance functionally important properties of the strips such as bioadhesive agents to ensure that the film adheres to the oral mucosal surface for the appropriate time periods in order to allow drug absorption. ${ }^{[8]}$

Various studies have been reported on the manufacture of oral strips using HME. Tumuluri et al., and Prodduturi et al., conducted extensive research on the use of HME for the manufacture of mucoadhesive buccal films with different matrix formers and additives. ${ }^{[12,13]}$ In an early investigation, the group found that the addition of plasticizers, such as triethyl citrate, PEG 2000/8000, or acetyltributyl citrate, allowed the manufacture of thin, flexible, and stable films when hydroxypropyl cellulose (HPC) was used as film forming polymer. ${ }^{[13]}$ It was shown that the increase in the molecular weight of HPC decreased the release of drugs from 
extruded films, which resulted in dissolution profiles exhibiting zero-order drug release. ${ }^{[14,15]}$

Ketoconazole (KTZ) is an antifungal agent mainly used for the treatment of local and systemic fungal infections in various forms (e.g. oral tablets, topical creams, gels, aerosols, suspensions and shampoos). Due to its very poor water solubility (17 $\mu \mathrm{g} / \mathrm{ml}$ ) and high permeability, KTZ is classified as Biopharmaceutics Classification System (BCS) class II drug. Increasing the solubility of KTZ is a challenging task as it significantly reduces the oral bioavailability of the drug and thus the therapeutic effectiveness. ${ }^{[15]}$ We report to the best of our knowledge, a new case to manufacture KTZ based oral strips using inorganic excipients via a continuous HME processing and the physicochemical characterisation of the developed strips where the novelty of this paper lies. The distribution of the drug particles on the surface of the manufactured oral strips is also investigated as well as the strips' hydration/swelling, mucoadhesion and mechanical properties.

\section{Materials and methods}

\section{Materials}

Ketoconazole (KTZ) was purchased from Sigma Aldrich (Gillingham, UK). Hydroxypropyl methylcellulose K4 (HPMC) and magnesium aluminometasilicate (Neusilin, MAS) were kindly donated by Colorcon (Dartford, UK) and Fuji Chemical Industry Co., Ltd. (Japan), respectively. Soluplus (SOL) was kindly donated by BASF, Germany. Sorbitol (SRB) and polyethylene glycol 2000 (PEG) were provided by Roquette (Lestrem, France) and Fluka (Gillingham, UK). Sodium CMC was purchased from Sigma Aldrich (Gillingham, UK). The HPLC solvents were of analytical grade and purchased from Fisher Chemicals (Loughborough, UK). All materials were used as received.

\section{Drug/polymer miscibility determination}

The drug/polymer miscibility was determined by calculating Hansen solubility parameters $(\boldsymbol{\delta})$ of both the drug and polymers. By considering the chemical structure and the orientations of all drug/polymers, the solubility parameters were calculated by using the following equation, which is known as Hoftyzer and Van Krevelen method: $[16,17]$ 


$$
\delta=\sqrt{\delta_{d}^{2}+\delta_{p}^{2}+\delta_{h}^{2}}
$$

Here,

$$
\delta_{d}=\frac{\sum F_{d i}}{V_{i}}, \delta_{p}=\frac{\sqrt{\sum F_{p i}^{2}}}{V_{i}}, \delta_{p}=\sqrt{\left(\sum E_{h i} / V_{i}\right)}
$$

Where $\delta$ is total solubility parameter calculated, $\mathrm{F}_{\mathrm{di}}$ is the constant (molar attraction) due to molar dispersion forces, $\mathrm{F}_{\mathrm{pi}}^{2}$ is the constant (molar attraction) due to molar polarization forces, $\mathrm{E}_{\mathrm{hi}}$ is the hydrogen bonding energy, and $\mathrm{V}_{\mathrm{i}}$ the group contribution to molar volume.

\section{Preparation of formulation blends and continuous HME processing}

KTZ formulations with HPMC and SOL as polymeric matrices and a drug loading ranging from $5-20 \%(\mathrm{w} / \mathrm{w})$ (Table 1$)$ were thoroughly mixed in a Turbula TF2 mixer (Basel, Switzerland) mixer in $100 \mathrm{~g}$ batches for $10 \mathrm{~min}$ each, prior to the extrusion process. About $1 \mathrm{~g}$ of powdered samples from each batch (physical mixture) was preserved in an air tight glass vial for further analyses. Appropriately weighted MAS, SRB and PEG were added to the Turbula mixtures prior to the extrusion process. Extrusion of all formulations were performed using a EuroLab 16 twin-screw extruder (Thermo Fisher, Germany) equipped with a specially designed die to produce strips of $0.5 \mathrm{~mm}$ thickness and temperature profile at $50^{\circ} \mathrm{C} / 130^{\circ} \mathrm{C} / 140^{\circ} \mathrm{C} / 140^{\circ} \mathrm{C} / 140^{\circ} \mathrm{C} / 140^{\circ} \mathrm{C} / 140^{\circ} \mathrm{C} / 140^{\circ} \mathrm{C} / 140^{\circ} \mathrm{C} / 140^{\circ} \mathrm{C}$ (from feeding zone $\rightarrow$ Die) temperature profiles. The screw speed maintained for all extrusions was 100 rpm. The extrudates were fed continuously to a PFL-590 cutting machine (Kunshan Pingfenglong Trade Co., Ltd, China) and cut into strips of $1 \mathrm{~cm} \times 5 \mathrm{~cm}$ (100 mg of $\mathrm{KTZ}$ in each strips).

\section{Hydration and swelling studies}

The hydration (swelling) profiles of the strips were determined using a modified method from that previously described. ${ }^{[18]}$ Briefly, the strips were initially weighed 
and the weight recorded as $W_{i}$ and then placed in a Petri dish containing $10 \mathrm{~mL}$ of 0.1 M PBS, pH $6.8 \pm 0.1$, at $37^{\circ} \mathrm{C}$. At pre-determined time intervals (20 minutes), the liquid medium was removed, the strips blotted carefully with tissue paper to remove the surface-adhered liquid droplets and reweighed on an electronic balance and recorded as $\mathrm{W}_{\mathrm{t}}$. Fresh medium $(10 \mathrm{ml})$ was then added to the already swollen strip and the process continued till maximum swelling was obtained (up to 80 minutes). The percentage of water uptake was calculated as follows:

$$
\text { Swelling Capacity }(\%)=100 X \frac{W_{t}-W_{i}}{W_{i}}
$$

Where, $W_{t}$ is the weight of the hydrated wafer at time $\mathrm{t}$, and $\mathrm{Wi}$ is the initial weight of wafer.

\section{In vitro mucoadhesion studies}

Mucoadhesive measurements were performed on the strips using a previously reported method. ${ }^{[19]}$ The strips ( $1 \mathrm{~cm} \mathrm{x} 2 \mathrm{~cm}$ dimensions) were attached to a $75 \mathrm{~mm}$ diameter adhesive rig probe with a double sided adhesive tape on a TA.HD.plus Texture Analyser (Stable Micro Systems, Surrey, UK) fitted with a $5 \mathrm{~kg}$ load cell in tension mode. The model mucosal surface was gelatin gel which was allowed to set in a Petri-dish (diameter $88 \mathrm{~mm}$ ). The set gelatin gel was equilibrated with $2 \%$ mucin solution (adjusted to $\mathrm{pH}$ of $6.8 \pm 0.1$ ) for $1 \mathrm{~min}$ and placed on the instrument platform. The probe, lined with the strip was lowered towards the gelatin surface at pre-test speed of $0.5 \mathrm{~mm} / \mathrm{s}$, test speed of $0.5 \mathrm{~mm} / \mathrm{s}$ and post speed of $1.00 \mathrm{~mm} / \mathrm{s}$. The maximum force required to detach the strip on the upper probe from the mucosal surface, known as the peak adhesion force (PAF) was determined. The area under the curve (AUC) representing total work of adhesion (TWA) of the wafers was estimated from the force-distance plot whiles the cohesiveness of the samples was determined by the distance of travel.

\section{Scanning electron microscopy (SEM)/ energy dispersive X-Ray (EDS) analysis}

The spatial distribution of discreet chemical phases was evaluated using a coldcathode field-emission gun scanning electron microscope (Hitachi SU8030 FEGSEM, Japan) and Thermo-Noran (USA) EDS system with $30 \mathrm{~mm}^{2}$ window Ultra-Dry detector and Noran 7 software. The samples were secured to aluminium stubs with 
Leit-C carbon cement and coated with carbon (Edwards 306 high vacuum carbon evaporation) before SEM/EDS analysis. The elemental distribution on the surface of the strips was investigated using EDS while surface analysis to characterize the morphology of strips was evaluated using SEM at nominal magnifications of x500 and $x 1000$. The accelerating voltage of the incident electron beam was set at $10 \mathrm{kV}$ in order to minimize beam damage to the sample while maintaining adequate excitation. The raw X-ray maps were processed for background removal to give net-counts for each of the analytical X-ray lines. Chlorine $(\mathrm{Cl})$ atoms were taken as a marker atom since it was only present in the structure of the drug.

\section{Thermal analysis}

The physical form of the pure drug, physical mixtures and extruded strips were examined by using a Mettler-Toledo 823e (Greifensee, Switzerland) differential scanning calorimeter. Samples were prepared in sealed aluminium pans (2-5 mg) with a pierced lid. The samples were heated at $10^{\circ} \mathrm{C} / \mathrm{min}$ under nitrogen atmosphere in a temperature range between $0^{\circ} \mathrm{C}$ and $250^{\circ} \mathrm{C}$.

\section{Transmission $\mathrm{X}$-ray diffraction analysis}

A D8 Advantage X-ray diffractometer (Bruker AXS GmbH, Karlsure, Germany) was employed to study the crystallinity of KTZ on the extruded strips. The transmission diffractograms were acquired with Diffrac plus XRD Commander over a start to end diffraction $2 \theta$ angle of $2-40^{\circ}$, scan speed of $0.2 \mathrm{~s}$ and step size of 0.02 and a counting time of 0.4 s per step; 176 channels active on the PSD making a total counting time of $70.4 \mathrm{~s}$ per step. The operating conditions were $40 \mathrm{kV}$ and $40 \mathrm{~mA}$ with $\mathrm{Cu} \mathrm{Ka}$ radiation. All acquired data were processed with EVA software for bulk substances and the extruded strips.

\section{In vitro drug release studies}

In vitro dissolution study of pure drug and extrudates was carried out by using a Varian 705 DS dissolution paddle apparatus (Varian Inc. North Carolina, US) at 100 $\mathrm{rpm}$ and $37 \pm 0.5^{\circ} \mathrm{C}$. The dissolution medium $\mathrm{pH}$ was maintained at 6.8 by using 900 $\mathrm{ml}$ of $0.2 \mathrm{M}$ potassium dihydrogen phosphate buffer (adjusted with $2.0 \mathrm{M} \mathrm{NaOH}$ solution) for $2 \mathrm{~h}$. The temperature was equilibrated to $37 \pm 0.5^{\circ} \mathrm{C}$. At predetermined 
time intervals, samples were withdrawn for HPLC assay and replaced with fresh dissolution medium. All dissolution studies were performed in triplicate.

\section{HPLC analysis}

The release of KTZ from the prepared extrudates (strips) was determined by HPLC. An Agilent Technologies system equipped with a Sperisorb S50DS1 (150 mm x 4.6 $\mathrm{mm} \times 5 \mu \mathrm{m}$ ) column at $230 \mathrm{~nm}$ was used for the KTZ HPLC assay. The mobile phase consisted of methanol/water/triethyl amine (80: 20:0.2, v/v/v). The flow rate was 1.5 $\mathrm{ml} / \mathrm{min}$ and the retention time of KTZ was about $2 \mathrm{~min}$. The KTZ calibration curve $\left(\mathrm{R}^{2}=0.999\right)$, at concentrations varying from $5 \mu \mathrm{g} / \mathrm{ml}$ to $100 \mu \mathrm{g} / \mathrm{ml}$, were used to evaluate all the samples with $20 \mu 1$ injection volume.

\section{Statistical analysis}

All statistical analysis of the data for swelling studies were conducted using Microsoft Excel 2013 (USA). Dissolution profiles were investigated for the significance of difference among the distribution of the independent variables using a non-parametric statistical test of significance - Kruskal-Wallis (Vassarstats, Poughkeepsie, NY USA).

\section{Results and discussion}

\section{Drug/polymer miscibility}

The prediction of drug/polymers miscibility prior to the selection of appropriate hydrophilic strips forming polymer in all extruded formulations was successfully determined by the solubility parameters $(\delta)$ using the Van Krevelen/ Hoftyzer equation 1. ${ }^{[17,20]}$ Generally, the drug/polymers miscibility is achieved via the optimised stability between the energy released by inter molecular interactions and intra molecular interactions within the components. ${ }^{[21]}$ The theoretical approach derived from the solubility parameter suggests that compounds with similar $\delta$ values are likely to be miscible. The reason is that the energy of mixing from intramolecular interactions with particular components is then balanced with the energy of mixing from intermolecular interactions between the components (drug/polymer). Therefore, it has been demonstrated that compounds with $\Delta \delta<7 \mathrm{MPa}^{1 / 2}$ are likely to be miscible and compounds with $\Delta \delta>10 \mathrm{MPa}^{1 / 2}$ are likely to be immiscible. ${ }^{[21,22]}$ 
The calculated solubility parameter of KTZ, HPMC and SOL were 20.60, 24.61 and $19.41 \mathrm{MPa}^{1 / 2}$, respectively. The difference between the calculated solubility parameters of the polymers and the drug indicates that KTZ is likely to form solid dispersions with both polymers HPMC and SOL. By using the Van Krevelen/Hoftyzer equation the $\Delta \delta$ values for KTZ/HPMC and KTZ/SOL were 4.01 and $1.19 \mathrm{MPa}^{1 / 2}$, respectively.

\section{Continuous manufacturing of strips via HME}

As shown in Figure 1 extrusion processing followed by downstream equipment was optimised to obtain the extrudates in the form of whitish strips. For the purposes of the study a specially designed die was coupled with the extruder to produce strips with approximately $0.5 \mathrm{~mm}$ thickness. The extruded strips were directed onto a compressed air facilitated conveyor belt and accurately cut into small pieces to produce strips of $1 \mathrm{~cm} \times 5 \mathrm{~cm}$ size. During the process optimisation of KTZ loaded strips, a range of temperatures profiles varying from $120^{\circ} \mathrm{C}-140^{\circ} \mathrm{C}$ feed rates and screw speeds were evaluated as they play a key role in the product development. The process was eventually optimized to provide a throughput of $1 \mathrm{Kg} / \mathrm{h}$. SOL was chosen due its ability to enhance the dissolution rates of poorly water-soluble drugs in HME processing while HPMC possess very good adhesive properties. ${ }^{[14]}$ In terms of the extrusion processibility, HPMC films appeared to be easier to handle compared to those of SOL due to the brittle nature of the latter. Therefore, the best two formulations (F1 and F2) with HPMC were taken for further characterisations The presence of MAS in the strip formulations facilitated smooth extrusion and subsequently cutting of the strips.

\section{Swelling capacity}

The hydration (swelling) profiles of the extruded films are presented in Table 2. The various formulations showed differences in their swelling profiles with the blank (no drug) HPMC and SOL strips showing slow initial hydration and gradual swelling over the measurement period. SOL containing strips generally showed significantly higher swelling $(\mathrm{p}<0.05)$ capacity than HPMC.

However, the KTZ containing strips showed very rapid hydration and swelling in the first $20 \mathrm{~min}$, significantly higher than blank strips followed by a drastic 
reduction after $40 \mathrm{~min}$ and eventually complete film disintegration occurred, suggesting it perhaps behaves as a semi-fast disintegrating system. This is due largely to the presence of MAS, which has high water affinity and hence cause rapid disintegration of the formulation. This is evidenced by the significantly very low swelling capacities recorded for the control strips in the absence of MAS. Further evaluation of the data shows that for blank formulations, peak swelling occurred at 60 min, whilst the drug loaded strips (F1 and F2) showed peak swelling capacity in only $20 \mathrm{~min}$ after which the values decreased significantly until they eventually disintegrated within a $60 \mathrm{~min}$ time period. This indicates that in addition to MAS, the amorphous nature of the drug present within the extruded strips contributed towards their initial hydration characteristics and eventual disintegration. The differences observed are interesting and expected to affect the dissolution profiles significantly since initial hydration and swelling, determine the rate of drug release by diffusion and/or eventual disintegration (erosion) of the polymer matrix.

\section{In vitro mucoadhesion studies}

Figure 2 shows the mucoadhesion (PAF, TWA and cohesiveness) profiles of the tested formulations. The HPMC based formulations showed higher PAF values than the SOL containing strips. In contrast, the drug loaded strips (F1 and F2) showed significantly $(\mathrm{p}<0.05)$ higher mucoadhesion (PAF and TWA) values compared to the blank strips, though the cohesiveness, which is the distance of travel before detachment, did not follow this trend, but it was still evident that the values were generally slightly higher for drug loaded strips than the blank ones. In addition, there was significant $(\mathrm{p}<0.05)$ difference in PAF and the cohesiveness between the higher KTZ (F1) and lower KTZ (F2) loaded strips whilst the TWA of the two formulations (F1 and F2) was almost identical. An interesting observation from the results is that F2 showed higher PAF and lower cohesiveness than F1 (opposite effect) even though both had similar TWA values. This is because the TWA is a function of both PAF and the distance travelled (area under the force-distance plot) and therefore their (PAF and cohesiveness) combined effect (TWA) was similar for both F1 and F2.

The above observations could be explained by the swelling capacity (Table 2), which plays an important role during the initial stages of mucoadhesion. In a typical mucoadhesion process the polymer hydrates initially which increases chain mobility. 
This subsequently allows the chains to interpenetrate into mucin present on the model mucosal surface and eventually form strong interactions via either hydrogen or covalent bonding. Therefore, the drug-loaded strips, which showed rapid hydration and subsequent disintegration (Table 2), will have exhibited a stronger initial interaction with the model mucosal surface. Further, because the volume of moisture available was not high on the gelatine surface (compared to oral mucosa washed with saliva), it had a longer time to bond more strongly with the model mucosal surface. However, due to its high apparent water affinity at lower drug loading (F2), the initial strong bonds are disrupted as the strips disintegrated to form slippery mucilage and therefore the cohesiveness will be expected to decrease.

Further, the control formulation (absence of MAS) showed higher PAF and TWA values than all the other formulations but lower cohesiveness than F1 and F2. Again, this could relate to the swelling and hydration behaviour as noted above, where the control strips showed very minimal hydration. This minimal hydration is enough to enable interaction with mucin but because the control strips did not hydrate to the same extent as F1 and F2, it will not have had the chance to travel over a longer distance before final detachment from the model mucosal substrate. Overall, it appears, that the main factors affecting the mucoadhesive properties were the drug content and the presence or absence of MAS in the formulations. Both the drug and MAS are not mucoadhesive and generally appear as brittle substances in formulations. The results also suggest that drug loaded strips will hydrate rapidly enough to allow drug diffusion out of the swollen gel but remain on the oral mucosa long enough to allow some level of absorption across the permeable oral (buccal and sublingual) mucosa.

\section{SEM/EDS analysis}

The surface morphology was examined by SEM using both secondary and backscattered electron imaging for both the drug and extruded strips (HPMC based). The strips containing HPMC polymer and excipients exhibited no drug crystals on the extrudate surface with KTZ (Figure 3). All extruded strips showed smooth surface. SEM/EDS was used to determine the elemental composition and distribution ${ }^{[23]}$ of KTZ strips. As can be seen in Figure 4a, C, O, Na, Mg, Al, Si and Cl atoms were detected on the surface of extruded films, which are directly related to the materials 
used during the HME processing. For example, the elemental distribution of $\mathrm{Mg}, \mathrm{Si}$ and $\mathrm{Al}$ indicates the presence of the synthetic amorphous MAS $\left(\mathrm{Mg} \cdot \mathrm{AlSiO} 3.2 \mathrm{H}_{2} \mathrm{O}\right)$ in the extruded strips. As expected, the most abundant element was carbon (which is present in a majority of the drugs and additives used in oral drug delivery) in both $\mathrm{KTZ}$ and all polymer/excipients used in this study.

Since none of the excipients/compounds, used during the extrusion process, contains $\mathrm{Cl}$ atoms in their chemical structures, it was treated as a marker atom. Therefore, any presence of $\mathrm{Cl}$ atoms in the extruded strips would have come from the drug itself. As can be seen in Fig. 4b that KTZ pure exhibits a homogenous distribution of $\mathrm{Cl}$ atoms in EDS mapping indicated by green color. Similarly, the distribution of KTZ on the surface of the strips was visualized by EDS elemental mapping of $\mathrm{Cl}$ atoms (Fig. 4b). Similar to the KTZ pure the distribution of $\mathrm{Cl}$ atoms on the surface of the continuously extruded strips was homogenous. Backscatter electron images (BEI) show the sample morphology, while the background subtracted net-count $\mathrm{Cl} \mathrm{K} \alpha \mathrm{X}$ ray maps and overlays of the X-ray maps onto the BEI show the homogenous distribution of $\mathrm{Cl}$ in the sample. From the EDS mapping analysis it is obvious that $\mathrm{KTZ}$ is homogenously dispersed into the manufactured strips during the extrusion processing.

\section{Thermal analysis}

Differential scanning calorimetry (DSC) was used to analyse the solid state of the pure API, polymers, their physical mixtures (PM) and active extruded strips (EXT). The overall findings from DSC results are summarized in Figures. 5a and $b$. The thermograms of pure KTZ showed an endothermic transition corresponding to its melting at $150.32^{\circ} \mathrm{C}(\Delta \mathrm{H}=98.78 \mathrm{~J} / \mathrm{g})$ with an onset at $147.88^{\circ} \mathrm{C}$. Similarly, the pure polymer showed a thermal transition via a clear step change at $158.76^{\circ} \mathrm{C}$ corresponding to the $\mathrm{Tg}$ of $\mathrm{HPMC}$ and $59.23^{\circ} \mathrm{C}$ corresponding to $\mathrm{Tg}$ of $\mathrm{NaCMC}$, respectively (Figure 5a). In addition, SRB and PEG exhibited sharp thermal transitions due the melting at 95.92 and $50.37^{\circ} \mathrm{C}$, respectively (Figure 5a) while amorphous MAS showed a flat line. The physical mixtures exhibited endothermic peaks (Figure 5a) corresponding to the initial substances at slightly shifted temperatures indicating the drug existence in its crystalline form. In the physical mixtures of F1 and F2, three endothermic peaks were visible (Figure 5b), one at 
$51.30^{\circ} \mathrm{C}$ corresponding to the melting peak of crystalline PEG present in the mixture followed by the transition at $98.52^{\circ} \mathrm{C}$ due to the melting of SRB. The endothermic peak at $150.25^{\circ} \mathrm{C}$ corresponds to the melting of the crystalline KTZ. Furthermore, the extruded formulations presented only one endothermic peak between 47.36 and $47.97^{\circ} \mathrm{C}$ that was attributed to SRB. The absence of melting endothermic KTZ peaks suggests the presence of the drug in amorphous state. ${ }^{[21,24,25]}$

\section{Transmission X-ray diffraction analysis}

The extruded strips, including pure drug and physical mixtures of the same composition as well as pure compounds were studied by transmission X-ray diffraction analysis in order to examine the crystalline state of the drug. As depicted in Figure $6 \mathrm{a}$ the diffractograms of pure KTZ presented distinct peaks at 6.75, 17.50, $19.08,20.42,23.79,24.42,28.22$ 29, while PEG showed two distinct peaks with relatively high intensity at 19.25 and 22.3120 . Similarly pure SRB showed its characteristic crystalline peaks at positions ranging from 11 to $35.202 \theta$. Due to their amorphous nature, HPMC and MAS showed typical amorphous halos in the diffractograms.

The physical mixture of both formulations presented identical peaks (data not shown) at lower intensities suggesting that the drug retain its crystalline properties. In the extruded strip formulations (Figure 6b) there were two distinct peaks that correspond to the presence of PEG, however no peaks due to the crystalline drug were observed. The absence of KTZ peaks strongly suggests the presence of amorphous API in the extruded solid dispersion containing oral strips.

\section{In vitro dissolution study}

The main challenge for this study was to increase the dissolution rate of the poorly water soluble KTZ (water solubility $17 \mu \mathrm{g} / \mathrm{ml}$ ) ${ }^{[15]}$ in order to provide a rapid release. In Fig. 7, it can be seen that extruded strips containing $20 \%$ of KTZ (F1) provided rapid dissolution rates (cumulative release of $70 \%$ in $60 \mathrm{~min}$ at $\mathrm{pH}$ 6.8) compared to bulk KTZ that presented significantly slower dissolution. The result was complemented by previous studies reported elsewhere and is expected given the $\mathrm{pKa}$ values for $\operatorname{KTZ}(2.94,6.15) .{ }^{[26]}$ This enhancement of the dissolution can be attributed 
to the formation of amorphous solid dispersions of KTZ in the extruded strips. In addition to the hydrophilic polymers, the presence of the inorganic MAS contributed to the enhanced release of KTZ. It has been reported that MAS releases silicic acid, $\mathrm{Mg}^{2+}$ and $\mathrm{Al}^{3+}{ }^{[27,28]}$ ions in dissolution medium, which facilitate the dissolution rates of poorly water-soluble drugs. The extruded strips presented similar release patterns and the $20 \% \mathrm{KTZ}$ loaded formulations provided slightly faster release rates compared to those of $15 \%(\mathrm{w} / \mathrm{w})$. Application of a single factor non-parametric statistical test of significance - Kruskal-Wallis test $(p>0.05)$ to the results showed no significant differences between the release profiles. Nevertheless, the HME extruded strips showed excellent release patterns that could provide fast onset action in future clinical trials.

\section{Conclusions}

HME was successfully employed to manufacture oral KTZ loaded strips in a continuous mode. EDS analysis revealed a homogeneous drug distribution on the surface of the extruded strips while thermal analysis and X-ray diffraction studies showed the presence of amorphous drug in the strips due to the intense mixing during the extrusion processing. The presence of KTZ in amorphous state facilitated enhanced in vitro dissolution rates at five orders of magnitude compared to the bulk drug substance. The use of MAS also favoured smooth processing of the extruded strips followed by rapid hydration, swelling and eventually enhanced dissolution rates of the poorly water-soluble ketoconazole.

\section{Conflict of interest}

The authors declare no conflict of interest

\section{Acknowledgements}

This research is sponsored by Fuji Chemical Industry Co. Ltd., Japan.

\section{References}

1. Morales JO et al. Films loaded with insulin-coated nanoparticles (ICNP) as potential platforms for peptide buccal delivery. Colloids and Surfaces B: Biointerfaces 2014; 122: 38-45. 
2. Giovino $\mathrm{C}$ et al. An integrated buccal delivery system combining chitosan films impregnated with peptide loaded PEG- $b$-PLA nanoparticles. Colloids and Surfaces B: Biointerfaces 2013; 112: 9-15.

3. Pongjanyakul T, Suksri H. Alginate-magnesium aluminum silicate films for buccal delivery of nicotine. Colloids and Surfaces B: Biointerfaces 2009; 74: 103-113.

4. Low AQ et al. Chan, Effect of type and ratio of solubilising polymer on characteristics of hot-melt extruded orodispersible films. Int J Pharm 2013; 15 455(1-2):138-47.

5. Barnhart S. Thin film oral dosage forms, in Rathbone MJ et al. (Eds.). Modified-release Drug Delivery Technology. Informa Healthcare 2008; 209 216.

6. International Conference on Harmonization, ICH topic Q3C (R3) Impurities: Residual Solvents, 2009. [http://www.emea.europa.eu/pdfs/human/ich/028395en.pdf]. Last Accessed on April 2016.

7. Morales JO, McConvill JT. Manufacture and characterization of mucoadhesive buccal films. Eur J Pharm Biopharm 2011; 77:187-99.

8. Repka MA et al. Encyclopedia of pharmaceutical technology, in J. Boylan J (ed.), Marcel Dekker, NewYork, 2002.

9. Jones DS et al. Characterisation and modelling of the thermorheological properties of pharmaceutical polymers and their blends using capillary rheometry: Implications for hot melt processing of dosage forms. Int $J$ Pharm 2015; 493(1-2):251-9.

10. Potter $\mathrm{C}$ et al. Novel supercritical carbon dioxide impregnation technique for the production of amorphous solid drug dispersions: a comparison to hot melt extrusion. Mol Pharm 2015; 12(5):1377-90.

11. Andrews GP, Jones DS. Hot melt extrusion - processing solid solutions. $J$ Pharm Pharmacol 2014; 66(2):145-7.

12. Tumuluri VS et al. Off-line and On-line Measurements of Drug-loaded HotMelt Extruded Films Using Raman Spectroscopy. Int J Pharm 2008; 357(1-2): 77-84.

13. Prodduturi $\mathrm{S}$ et al. Solid-state stability and characterization of hot-melt extruded poly(ethylene oxide) films. J Pharm Sci 2005; 94: 2232-2245. 
14. Francesco C et al. Fast dissolving films made of maltodextrins. Eur J Pharm Biopharm 2008; 70: 895-900.

15. Karolewicz B et al. Thermal, spectroscopic, and dissolution studies of ketoconazole-Pluronic F127 system. J Therm. Anal Calorim 2014; 115: 24872493.

16. Hansen CM. The universality of the solubility parameter. Ind Eng Chem Res Dev 1969; $8: 2-11$.

17. Hoftyzer PJ, Krevelen DWV. Properties of copolymers. Amsterdam: Elsevier, 1976.

18. Kianfar F et al. Development and physico-mechanical characterization of carrageenan and poloxamer based lyophilized $\mathrm{p}$ matrix as a potential buccal drug delivery system. Drug Dev Ind Pharm 2014; 40(3): 361-369.

19. Ayensu I et al. Effect of membrane dialysis on characteristics of lyophilised chitosan wafers for potential buccal delivery of proteins. Int J Bio Macro 2012; 50: 905-909.

20. Maniruzzaman M et al. Drug-copolymer intermolecular interactions in hotmelt extruded solid dispersions. Int J Pharm 2013; 443(1-2): 199-208.

21. Maniruzzaman M et al. Dissolution enhancement of poorly water-soluble APIs processed by hot-melt extrusion using hydrophilic copolymers. Drug Dev Ind Pharm 2013; 39(2): 218-27.

22. Maniruzzaman M et al. Taste masking of paracetamol by hot-melt extrusion: an in vitro and in vivo evaluation. Eur J Pharm Biopharm 2012; 80(2): 433-42.

23. Vithani K et al. Sustained release solid lipid matrices processed by hot-melt extrusion (HME). Colloids Surf B Biointerfaces 2013; 110: 403-10.

24. Zheng $X$ et al. Part I: Characterization of Solid Dispersions of Nimodipine Prepared by Hot-melt Extrusion. Drug Dev Ind Pharm 2007; 33: 791-802.

25. Grycze A et al. Development and evaluation of orally disintegrating tablets (ODTs) containing ibuprofen granules prepared by hot melt extrusion. Colloids Surf B Biointerface 2011; 86: 275-84.

26. Zhou $\mathrm{R}$ et al. pH-Dependent Dissolution in vitro and absorption in vivo of weakly basic drugs: development of a canine model. Pharm Res 2005; 22: 188192. 
27. Bahl D and Bogner RH. Amorphization of Indomethacin by Co-Grinding with Neusilin US2: Amorphization Kinetics, Physical Stability and Mechanism. Pharm Res 2006; 23: 2317-25.

28. Bahl D and Bogner RH. Amorphization Alone Does Not Account for the Enhancement of Solubility of Drug Co-ground with Silicate: The Case of Indomethacin. AAPS PharmSciTech 2008; 9: 146-153.

\section{TABLES}

Table 1: Formulation composition of the KTZ oral strips (w/w \%)

\begin{tabular}{|lccccccc|}
\hline Excipients & F1 & F2 & F3 & F4 & $\begin{array}{c}\text { HPMC } \\
\text { (Blank) }\end{array}$ & $\begin{array}{c}\text { SOL } \\
\text { (Blank) }\end{array}$ & Control \\
\hline KTZ & 20 & 15 & 20 & 15 & - & - & - \\
HPMC & 30 & 40 & - & - & 50 & - & 60 \\
SOL & - & - & 30 & 40 & - & 50 & - \\
MAS & 10 & 10 & 10 & 10 & 10 & 10 & - \\
PEG & 20 & 15 & 20 & 15 & 20 & 20 & 20 \\
SRB & 10 & 10 & 10 & 10 & 10 & 10 & 10 \\
NaCMC & 10 & 10 & 10 & 10 & 10 & 10 & 10 \\
\hline
\end{tabular}

Table 2: Swelling profiles for the different formulations tested showing differences in hydration behaviour based on type of polymer and drug content

\begin{tabular}{|l|cccc|}
\hline \multirow{2}{*}{ Formulation } & \multicolumn{5}{|c|}{$\begin{array}{c}\text { Swelling (\%) } \\
\text { Time (min) }\end{array}$} \\
\cline { 2 - 5 } & 20 & 40 & 60 & 80 \\
\hline HPMC (Blank) & $203.53 \pm 0.30$ & $232.69 \pm 0.25$ & $239.74 \pm 0.30$ & $226.60 \pm 0.1$ \\
SOL (Blank) & $292.73 \pm 0.25$ & $413.94 \pm 0.33$ & $427.27 \pm 0.30$ & $418.79 \pm 0.2$ \\
Control & $4.41 \pm 0.31$ & $5.25 \pm 0.30$ & $5.09 \pm 0.20$ & $3.65 \pm 0.20$ \\
F1 & $509.58 \pm 0.35$ & $395.02 \pm 0.30$ & Disintegrated \\
F2 & $408.70 \pm 0.30$ & $263.35 \pm 0.20$ & Disintegrated \\
\hline
\end{tabular}




\section{Figures caption list}

Figure 1 i) Continuous manufacturing process of oral KTZ strips via advanced HME process, ii) digital photographs of representative extruded KTZ strips.

Figure 2 In vitro mucoadhesion data for the various blank and drug loaded formulations showing changes in mucoadhesive properties with change of polymer and drug loading content.

Figure 3 SEM images of KTZ loaded oral strips (F1).

Figure 4 a) Elemental analysis mapping of the extruded strips using EDS (F2) and b) EDS mapping of the $\mathrm{Cl}$ atoms (marker atom) of both $\mathrm{KTZ}$ and extruded strips to analyse the drug distribution on the surface of the continuously extruded strips.

Figure 5 DSC thermograms of a) bulk KTZ, MAS, PEG, SRB, HPMC, $\mathrm{NaCMC}$ and $\mathrm{b}$ ) extruded strips (EXT) and physical mixtures.

Figure 6 XRD-transmission diffractograms of a) pure substances (MAS, SRB, HPMC, KTZ and PEG) and b) extruded strips (F1 and F2) and bulk KTZ.

Figure 7 In vitro dissolution analysis of $\mathrm{KTZ}$ and extruded strips in phosphate buffer $\mathrm{pH} 6.8\left(\mathrm{n}=3,37 \pm 0.5^{\circ} \mathrm{C} ; 100 \mathrm{rpm}\right)$. 


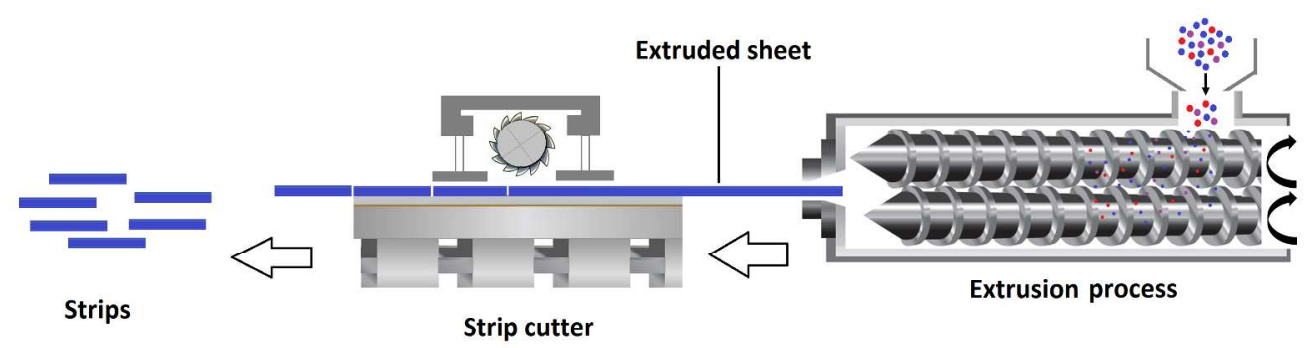

(i)

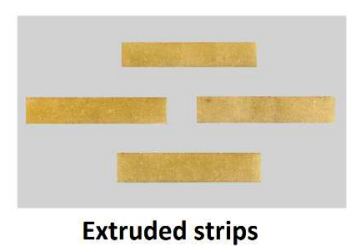

(ii)

Fig. 1

$779 \times 442 \mathrm{~mm}(96 \times 96$ DPI) 

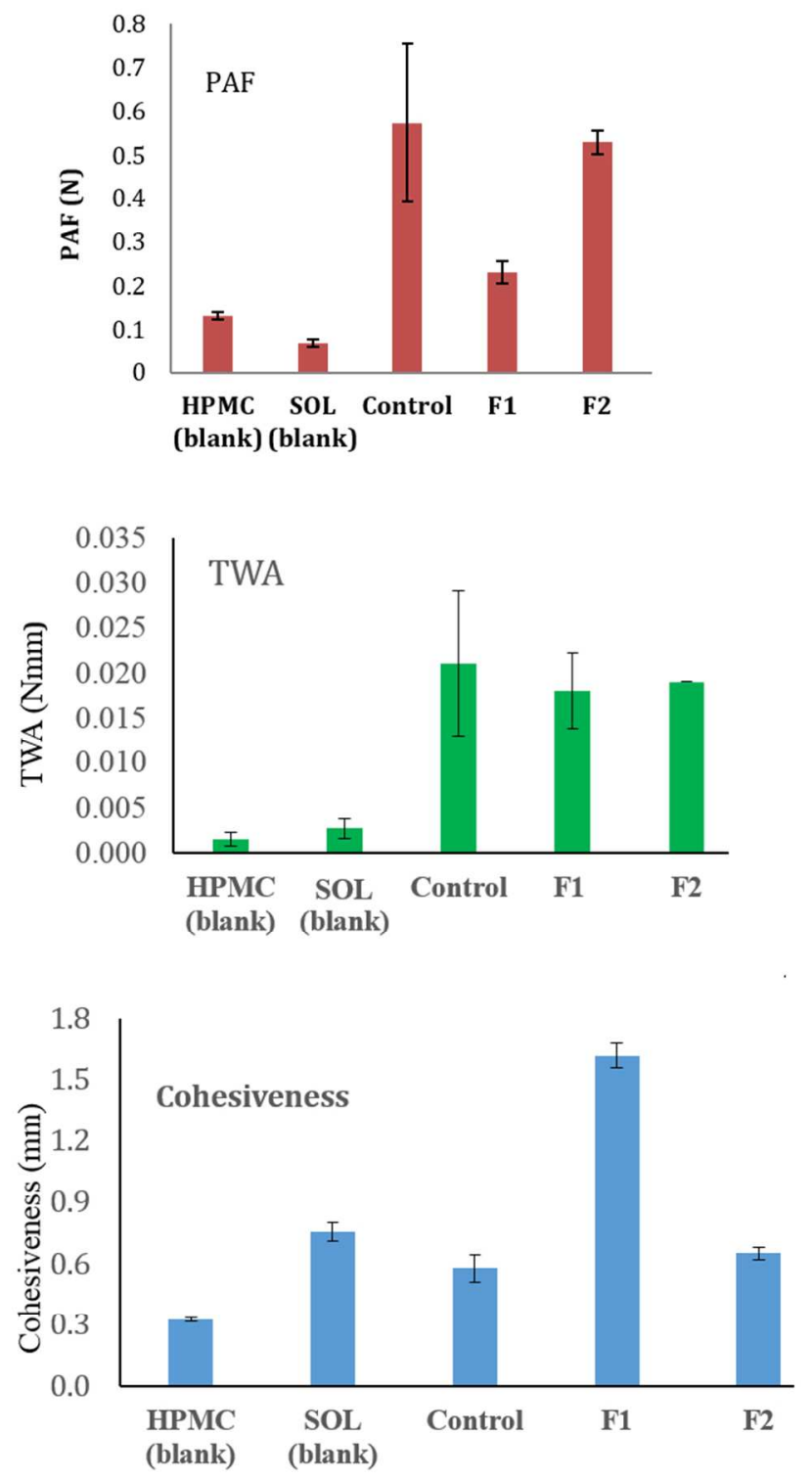

Fig. 2

$176 \times 327 \mathrm{~mm}(96 \times 96 \mathrm{DPI})$ 


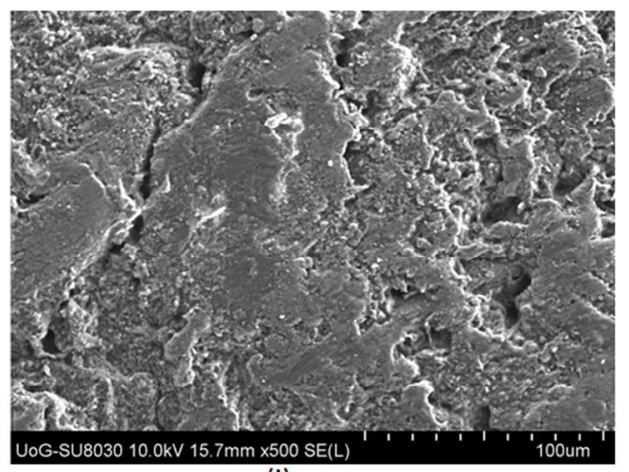

(i)

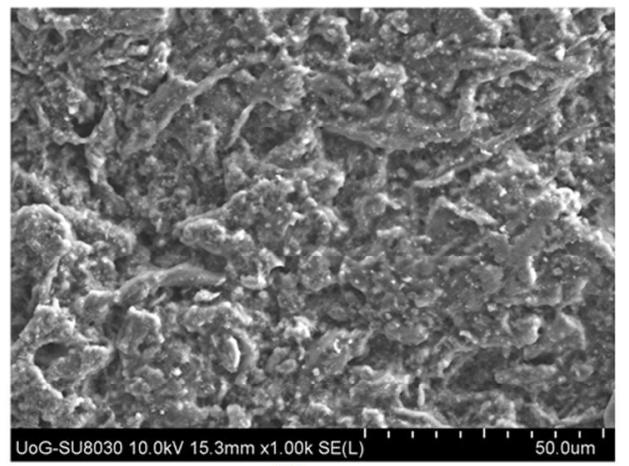

(ii)

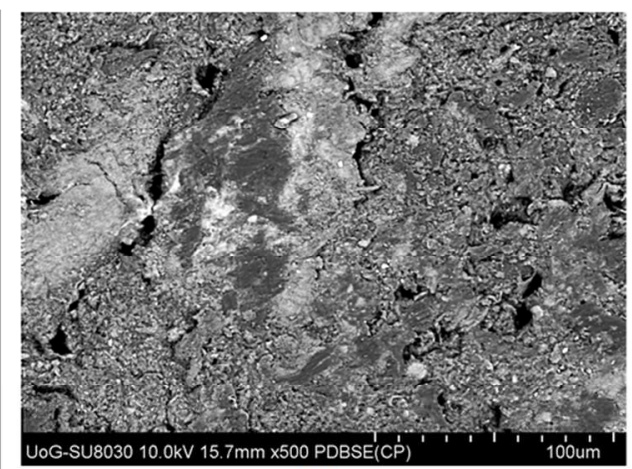

(iii)

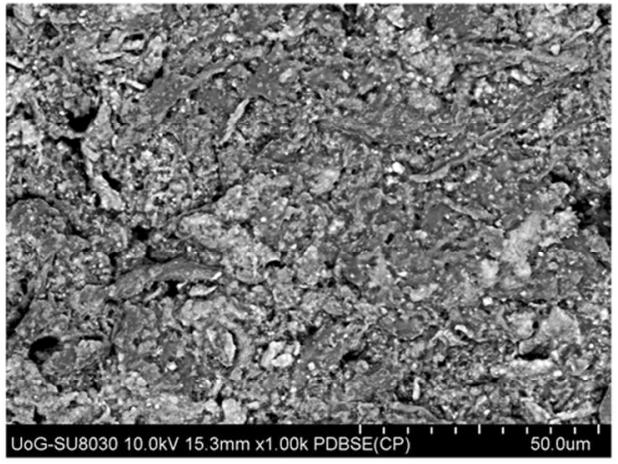

(iv)

Fig. 3

$277 \times 218 \mathrm{~mm}(96 \times 96 \mathrm{DPI})$ 

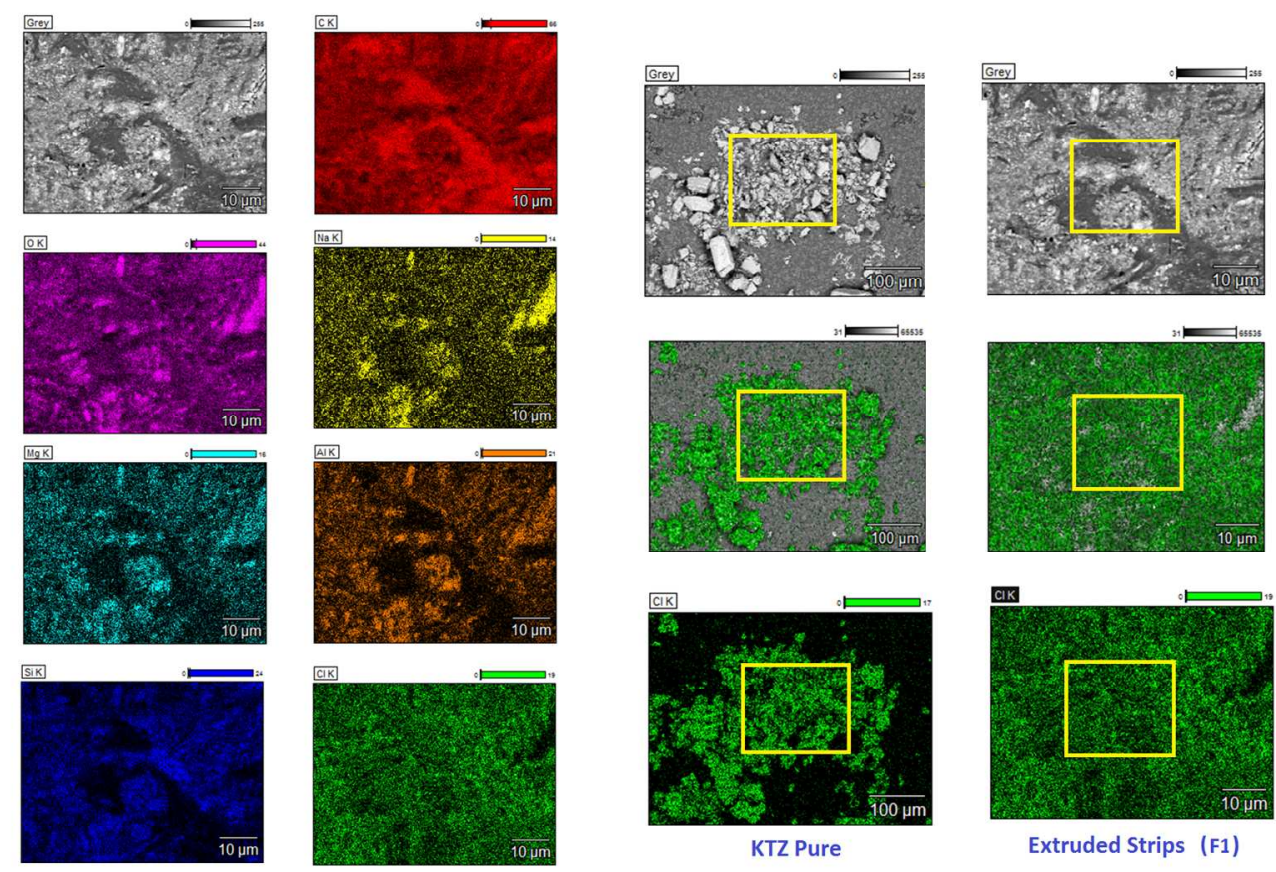

Extruded Strips (F1)

(a)

(b)

Fig. 4

$442 \times 324 \mathrm{~mm}(96 \times 96$ DPI) 


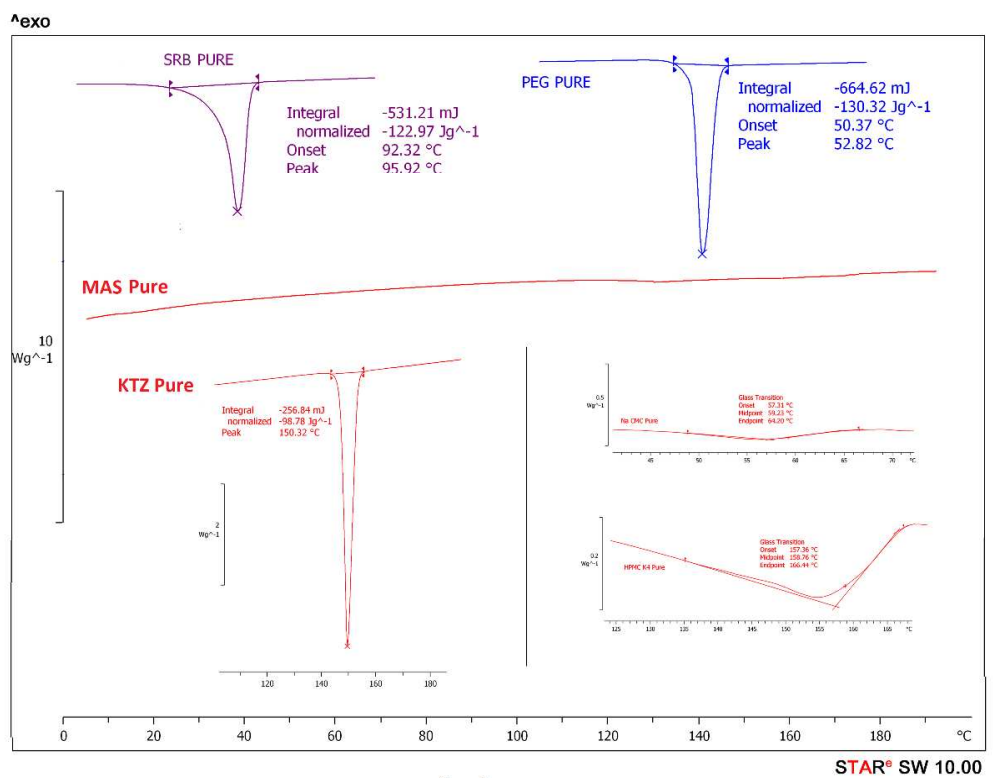

(a)

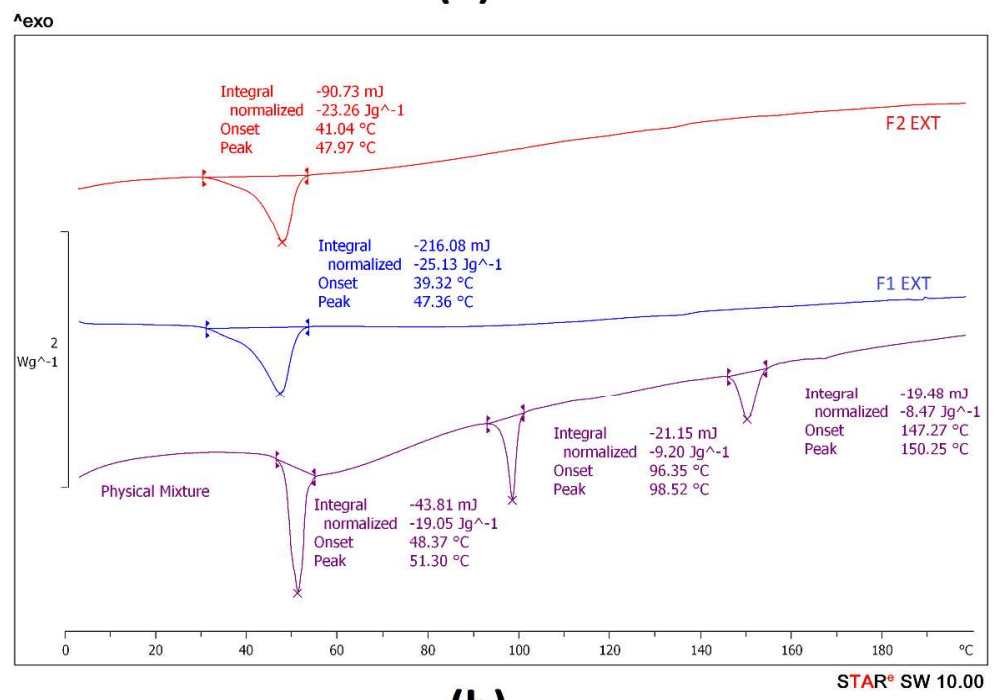

(b)

Fig. 5

$819 \times 1272 \mathrm{~mm}(96 \times 96$ DPI) 
Page 25 of 25

Journal of Pharmacy and Pharmacology

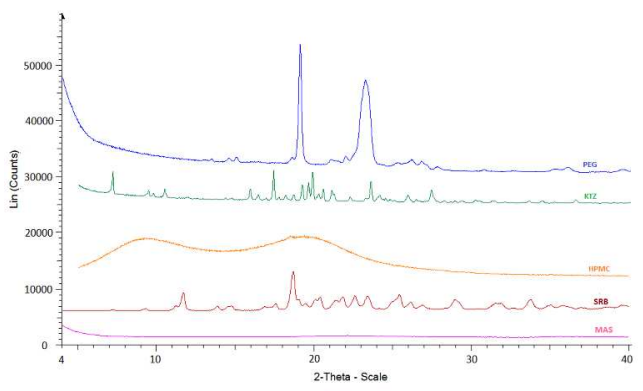

(a)

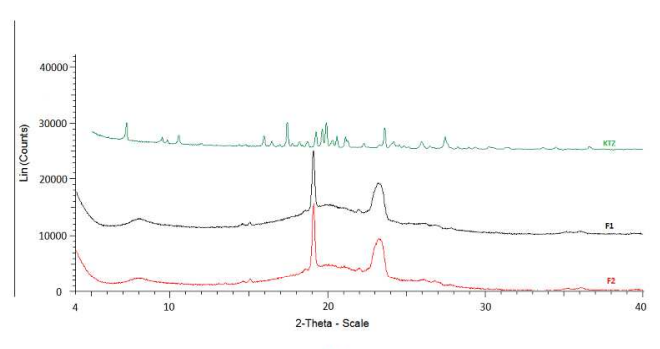

(b)

Fig. 6

$488 \times 162 \mathrm{~mm}(120 \times 120 \mathrm{DPI})$ 


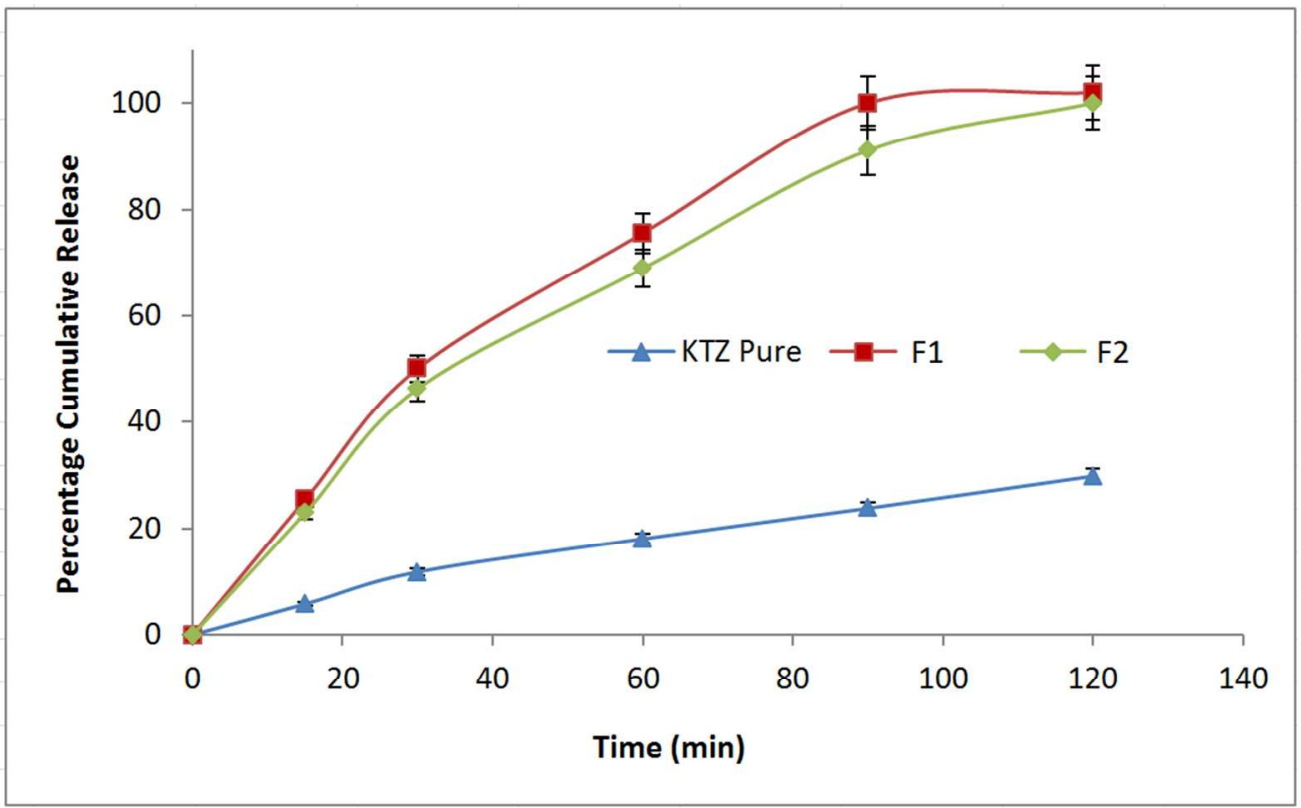

Fig. 7

$286 \times 177 m m(96 \times 96$ DPI $)$ 